\title{
ÜBER EINE KLASSE VON L-FUNKTIONEN ALGEBRAISCHER ZAHLKÖRPER
}

\author{
S.-N. KURODA
}

In der vorliegenden Arbeit konstruieren wir, indem wir die Schlüsse von Armitage [1] in einer für unseren Zweck geeigneten Form erbringen, eine andere Klasse von $L$-Funktionen als bei Armitage [1] und Fröhlich [5], welche an der Stelle $s=1 / 2$ eine Nullstelle ungerader Ordnung haben. ${ }^{1)}$

Es sei $G$ eine endliche Gruppe und $H$ eine von der Einsgruppe $E$ verschiedene echte Untergruppe von $G$. $G$ heißt eine Frobeniusgruppe zu $H$, wenn $H \cap H^{\sigma}=E$ gilt für alle $\sigma$ aus $G-H$, wo $H^{\sigma}=\sigma^{-1} H \sigma$ ist. Die Menge $F=G-\cup_{\sigma \in G}(H-E)^{\circ}$ bildet dann bekanntlich einen Normalteiler von $G . \quad F$ heißt der Frobeniuskern von $G .{ }^{2}{ }^{2}$

Um unseren Satz einzuführen, sei zunächst $k$ ein algebraischer Zahlkörper von endlichem Grade und $L / k$ ein galoisscher Körper, dessen Galoisgruppe $G$ eine Frobeniusgruppe ist. Wir bezeichnen mit $K$ den Invariantenkörper von $F$. Ist $\psi$ ein Charakter vom Grade 1 von der absoluten Idealklassengruppe von $K$, so sehen wir $\psi$ auch als Charakter der Galoisgruppe eines geeignet groß gewählten galoisschen Körpers über $K$ an, welcher den $\psi$ zugeordneten Klassenkörper enthält. Ist ferner der Wertvorrat von $\psi$ gleich $\{ \pm 1\}$, so nennen wir $\psi$ einen unverzweigten, quadratischen Charakter von $K$.

Es bezeichne nun $h\left(F^{\prime}\right)$ die Anzahl der Konjugiertenklasse von der Gruppe $F$ und $|H|$ die Ordnung von $H$. Dann läßt sich die $h(F)-1$ irreduziblen, nichttrivialen Charaktere von $F$ in die $n=(h(F)-1) /|H|$

Received March 30, 1974.

1) Vgl. auch Serre [13]. In [6] hat Fröhlich angedeutet, daß es für einen beliebigen algebraischen Zahlkörper unendlich viele galoissche Erweiterungen gibt mit der verallgemeinerten Quaternionengruppe als Galoisgruppe, deren Zetafunktionen an der genannten Stelle verschwinden. Im folgenden beschäftigen wir uns mit dem Fall, wo die Galoisgruppe eine Frobeniusgruppe ist.

2) Vgl. Huppert [8], Kapitel V, §8. Im folgenden werden wir für die benötigen Ergebnisse der Frobeniusgruppe auf die einschlägigen Paragraphen von [8] verweisen. 
Klassen einteilen, welche je aus denjenigen $|H|$ Charakteren bestehen, die auf $G$ denselben irreduziblen Charakter induzieren (vgl. [8], Kapitel $\mathrm{V}, \S 16.13)$. Wir numerieren die irreduziblen, nichttrivialen Charaktere von $F$ so, daß die ersten $\chi_{\nu}$ mit $1 \leqq \nu \leqq n$ diese $n$ Klassen der irreduziblen, nichttrivialen Charaktere von $F$ vertreten. Man setze nunmehr

$$
\chi=\sum_{\nu=1}^{n} \chi_{\nu}(1) \chi_{\nu}
$$

SATZ 1. Es sei L/k ein galoisscher Zahlkörper, dessen Galoisgruppe $G$ eine Frobeniusgruppe mit dem Frobeniuskern $F$ ist. Den Invariantenkörper von $F$ bezeichnen wir mit $K$. Wir nehmen an, daß jede unverzweigte, quadratische Erweiterung von $K$ galoissch über $k$ ist. Gehört der mit dem Charakter (1) gebildete Artinsche Führer

$$
\mathfrak{f}=\mathfrak{f}(\chi, L / K)
$$

keiner Quadratklasse (von der absoluten Idealklassengruppe) von $K$ an, dann gibt es einen unverzweigten, quadratischen Charakter \& von $K$ mit $\psi(\mathfrak{f})=-1$ derart, daß die mit dem Charakter $\psi \chi$ gebildete L-Funktion

$$
L(s, \psi \chi)
$$

an der Stelle $s=1 / 2$ eine Nullstelle ungerader Ordnung hat.

Nach der Funktionalgleichung der L-Funktion folgt dann das folgende Korollar.

Korollar. Unter denselben Voraussetzungen wie in Satz 1 ist die bei der Funktionalgleichung der L-Funktion (3) auftretende Konstante $W(\psi \chi)$ gleich -1 .

Es gibt die Fälle, wo sich die in Satz 1 von $f(\chi, L / K)$ geforderte Voraussetzung in Worten vom rationalen Zahlkörper $\boldsymbol{Q}$ charakterisieren läßt. Einen Spezailfall, wo auch in (1) $n=1$ gilt, wollen wir als Satz hevorheben.

SATZ 2. Es sei $q$ eine Primzahl mit $q \equiv 1(\bmod 12)$. Man setze $k=Q(\sqrt{-3 q})$ und $K=k(\omega)=Q(\sqrt{-3 q}, \sqrt{-3})$, wo $\omega$ eine primitive 3-te Einheitswurzel bedeutet. (Dann gibt es genau einen unverzweigten, quadratischen Charakter von $K$, wie nachträglich beim Beweis von Satz 
2 bemerkt wird.) Es sei angenommen, daß die Klassenzahl von $k$ zu 3 prim ist. Es bezeichne p eine Primzahl mit

$$
\left(\frac{\dot{q}}{p}\right)=\left(\frac{-3}{p}\right)=-1
$$

wobei die Klammer das Kroneckersche Symbol bedeutet. Wir bezeichnen mit $\mathfrak{p}$ einen Primteiler von $p$ in $k$. Dann gibt es eine nichtnegative, ganzrationale Zahl e derart, daß das Ideal $(1-\omega)^{e} \mathfrak{p}$ in $K$ Führer eines relativzyklischen kubischen Körpers $L / K$ ist, welcher sogar über $k$ galoissch ist. Wir bezeichnen mit $\chi$ einen dem Klassenkörper $L / K$ zugeordneten Charakter von $K$.

Alsdann hat die mit dem bikubischen Charakter $\psi \chi$ gebildete LFunktion $L(s, \psi \chi)$ an der Stelle $s=1 / 2$ eine Nullstelle ungerader Ordnung und gilt auch $W(\psi \chi)=-1$.

Die in (4) aufgestellte Bedingung ist gerade diese, die besagt, daß der Zerlegungskörper bezüglich $K / \boldsymbol{Q}$ einer rationalen Primzahl $p$ der Köper $k$ ist.

Im Fall, wo die Klassenzahl von $k$ durch 3 teilbar ist, haben wir in Satz 2 nur noch die Bedingung hinzuzufügen, daß p äquivalent der 3ten Potenz eines Ideals in $k$ ist.

Bemerkung. Im Beweis von Satz 1 spielt der fundamentale Satz von Thompson [14], daß der Frobeniuskern nilpotent ist, eine wichtige Rolle. Wir stellen eine hierzugehörige Folgerung beim Beweis von Satz 1. Diese gilt ohne die gemachten Annahmen über die Klasse von $\mathrm{f}(\chi, L / K)$ und das Verhalten über $k$ von unverzweigten, quadratischen Erweiterungen von $K$.

FOLGERUNG. Es bezeichne \& einen klassenkörpertheoretischen Charakter von $K$. Stimmt der Durchschnitt von $L$ und dem $\psi$ zugeordneten Klassenkörper mit $K$ überein, so ist $L(s, \psi \chi)$ eine ganze Funktion. Insbesondere ist also $L(s, \chi)$ eine ganze Funktion.

Nach Formel (6) unten ist der Quotient von der Zetafunktion des Invariantenkörpers zu $H$ durch diese von $k$ gleich $L(s, \chi)$. Daher ist der genannte Quotient eine ganze Funktion. Dies ist von Ishida [9] in Anlehnung an die Methode von Brauer [3] bewiesen. 


\section{§1. Beweis von Satz 1.}

1.1. Um die Schlüsse von Armitage [1] in einer für unseren Zweck geeigneten Form zu formulieren, sei zunächst $G$ eine beliebige endliche Gruppe und $L / k$ ein galoisscher Zahlkörper mit der Galoisgruppe $G$. Es sei nun $\chi$ ein Charakter von $G$ und $\bar{\chi}$ der komplex-konjugierte von $\chi$. Die Funktionalgleichung der Artinschen $L$-Funktion $L(s, \chi)$ nimmt die folgende Gestalt an :3)

$$
M(s, \chi)=W(\chi) M(1-s, \bar{\chi}),
$$

wobei der Quotient $M(s, \chi) / L(s, \chi)$ aus den bekannten Exponential- und $\Gamma$-Faktoren besteht und bei $s=1 / 2$ holomorph und von Null verschieden ist, während $W(\chi)$ eine Konstante ist vom absoluten Betrag 1 .

Es sei nun $H$ ein Normalteiler von $G$, und $K$ bezeichne den Invariantenkörper von $H$. Die Behauptung des folgenden Lemmas hat Armitage a.a.O. im Fall bewiesen, wo $G=H$ ist (vgl. [1], S. 202, Proposition 2).

LEMMA 1.1. Wir nehmen an, daß jede unverzweigte, quadratische Erweiterung von $K$ galoissch $\ddot{\text { über }} k$ ist. Es sei hier $\chi$ ein Charakter von $H$ und $\chi^{G}$ der durch $\chi$ induzierte Charakter von G. Man setze $\mathfrak{f}=\mathfrak{f}(\chi, L / K)$. Ist $\chi^{G}$ reellwertig, d.h. wenn $\chi^{G}(\sigma)$ für alle $\sigma$ aus $G$ reell ist, so sind die folgenden beiden Aussagen äquivalent.

a) Es gibt einen unverzweigten, quadratischen Charakter $\psi$ von $K$ mit $\psi(\mathfrak{f})=-1$ derart, daß das Produkt $L(s, \chi) L(s, \psi \chi)$ an der Stelle $s=1 / 2$ eine Nullstelle oder einen Pol ungerader Ordnung hat.

b) Der Führer $\mathrm{f}(\chi, L / K)$ gehört keiner Quadratklasse von $K$ an.

Zum Beweis von Lemma 1.1 können wir ohne Beschränkung der Allgemeinheit annehmen, daß die galoissche Erweiterung $L / K$ von vornherein so groß gewählt ist, daß sie alle unverzweigten, quadratischen Erweiterungen von $K$-wenn überhaupt diese existieren - umfassen. Dann folgt aus der Voraussetzung, daß der Kern von $\psi$ ein Normalteiler von $G$ ist. Daher läßt sich $\psi$ als Klassenfunktion auf $G$ erweitern, indem man etwa $\psi(\sigma)=0$ setzt für $\sigma$ aus $G-H$. Aus der bekannten Formel ${ }^{4)}$ $(\chi \cdot \operatorname{Res} \psi)^{G}=\chi^{G} \cdot \psi$ folgt dann, daß $(\psi \cdot \chi)^{G}$ auch reellwertig ist, wenn $\chi^{G}$ es ist.

Nun sei $M(s, \chi)$, wie oben, die $\chi$ zugehörige erweiterte $L$-Funktion.

3) Vgl. Artin [2] oder Hasse [7].

4) Vgl. Serre [12], §7. 
Dann gilt bekanntlich die allgemeine Regel $M\left(s, \chi^{\theta}\right)=M(s, \chi)$, aus der auch $W(\chi)=W\left(\chi^{G}\right)$ folgt. Ferner gilt dann unter unserer Voraussetzung $M(s, \bar{\psi} \chi)=M\left(s,(\psi \chi)^{G}\right)=M(s, \psi \chi)$. Daher haben wir

$$
\left\{\begin{array}{rl}
M(s, \chi) & =W(\chi) M(1-s, \chi) \\
M(s, \psi \chi) & =W(\psi \chi) M(1-s, \psi \chi)
\end{array} .\right.
$$

Ersetzt man $s$ durch $1-s$, so erhält man beispielsweise $M(1-s, \chi)=$ $W(\chi) M(s, \chi)$. Folglich erhält man $W(\chi)^{2}=W(\psi \chi)^{2}=1$.

Da nun, wie in Armitage [1] bestätigt ist, die Äquivalenz der beiden Aussagen a) und b) eine Konsequenz dieser Art von den Funktionalgleichungen (5) ist, ist der Beweis von Lemma 1.1 auf diesen bei Armitage a.a.O. zurückgeführt.

1.2. Nun kehren wir zurück auf den Fall, wo die Galoisgruppe $G$ eines galoisschen Zahlkörpers eine Frobeniusgruppe zu $H$ mit dem Frobeniuskern $F$ ist. Es bezeichne $h(H)$ die Anzahl der Konjugiertenklassen von $H$. Dann hat $G$ ausser den schon genannten $n$ irreduziblen Charakteren $\chi_{\nu}{ }^{G}(1 \leqq \nu \leqq n)$ nur noch diejenigen $\mu_{\eta}(1 \leqq \eta \leqq h(H))$, welche auf $H$, wenn diese auf $H$ beschränkt werden, die $h(H)$ irreduziblen Charaktere liefern und $\mu_{\eta}(\sigma \tau)=\mu_{\eta}(\sigma)$ genügen für alle $\sigma$ und $\tau$ aus $H$ bzw. $F$ (vgl. [8], Kapitel V, § 16.13).

Wir bezeichnen mit $1_{H}$ und $1_{G}$ die trivialen Charaktere von $H$ bzw. $G$ und mit $1_{H}^{G}$ den durch $1_{H}$ induzierten Charakter von $G$. Dieser liefert auf $F$ den Charakter der regulären Darstellung von $F$. Bezeichnet man ferner mit $\chi^{G}$ den durch den in (1) definierten Charakter $\chi$ von $F$ induzierten Charakter von $G$, so erhält man die folgende Charakterrelation $^{5)}$, indem man die Werte der beiden Seite für die Elemente aus $H$ bzw. $F$ vergleicht:

$$
\mathbf{1}_{H}^{G}=\mathbf{1}_{G}+\chi^{G} .
$$

Geht man auf die Beziehung von $L$-Funktionen über, so erhält

$$
\zeta(s, \Omega)=\zeta(s, k) L\left(s, \chi^{G}\right)=\zeta(s, k) L(s, \chi),
$$

wobei $\zeta(s, \Omega)$ und $\zeta(s, k)$ die Zetafunktionen vom Invariantenkörper $\Omega$ zu $H$ bzw. von $k$ bezeichnen. Da die bei der Funktionalgleichung der Zetafunktionen auftretende Konstante gleich 1 ist, hat bekanntlich die

5) Formel (6) stimmt inhaltlich überein mit der Formel (10) bei Ishida [9], die durch Spezialisierung der Brauerschen Charakterrelation [3] hergeleitet ist. 
Zetafunktion an der Stelle $s=1 / 2$ eine gerade Ordnung. Nach (7) hat folglich $L(s, \chi)$ an derselben Stelle eine gerade Ordnung.

Gilt nun die Voraussetzung in Satz 1, so hat nach Lemma.1.1 die Funktion $L(s, \chi) L(s, \psi \chi)$ an der Stelle $s=1 / 2$ eine Nullstelle oder einen Pol ungerader Ordnung. In diesem Fall hat folglich $L(s, \psi \chi)$ selbst an der genannten Stelle eine Nullstelle oder einen Pol ungerader Ordnung.

Wir haben noch nur zu zeigen, daß $L(s, \psi \chi)$ bei $s=1 / 2$ holomorph ist. Nach einem fundamentalen Satz von Thompson ist aber der Frobeniuskern nilpotent (vgl. [14] oder [8], S. 499). Dann ist auch die Galoisgruppe bezüglich $K$ des durch $L / K$ und den $\psi$ zugeordneten quadratischen Körper über $K$ zusammengesetzten Körpers nilpotent. Die Galoisgruppe dieses letztens genannten hat somit die Eigenschaft, daß jeder Charakter derselben eine positive, ganzrationale Linearkombination aus denjenigen Charakteren ist, die von gewissen Charakteren ersten Grades von Untergruppen induziert sind. Daher läßt sich $L(s, \psi \chi)$ als Potenzprodukt mit positiven, ganzrationalen Exponenten von gewissen mit klassenkörpertheoretischen Charakteren gebildeten $L$-Funktionen ausdrücken. Nach Hecke ist also $L(s, \psi \chi)$ bis auf die Stelle $s=1$ holomorph.

Zusammen mit dem oben Gesagten ist der Beweis von Satz 1 beendet.

Um die früher erwähnte Folgerung beim Beweis von Satz $1 \mathrm{zu}$ bestätigen, hat man nur noch das folgende $\mathrm{zu}$ beachten. Unter der Voraussetzung der genannten Folgerung kommt der triviale Charakter in $\psi \chi$ nicht vor. Damit kann man $\psi \chi$ als eine positive, ganzrationale Linearkombination aus denjenigen Charakteren ausdrücken, die durch gewisse nichttriviale Charaktere ersten Grades von Untergruppen induziert sind. Geht man nun auf die Beziehung der $L$-Funktionen über, so erhält man, wie oben, die zu beweisende Behauptung.

\section{§ 2. Beweis von Satz 2.}

2.1. Bezeichnungen und Voraussetzungen seien wie in Satz 2. Ferner bezeichnen wir mit $\mathfrak{\Re}_{k}$ die Idealklassengruppe von $k$, deren Hauptklasse aus denjenigen Idealen $\mathfrak{a}$ besteht, für welche $\mathfrak{a}^{m}=(\alpha), \alpha \in k$, gilt mit einem zu 2 primen geeigneten Exponenten $m . \Re_{k}$ heißt die 2Klassengruppe von $k$. Ebenso bezeichnen wir mit $\Re_{K}$ die 2-Klassengruppe von $K$. Die Ordnungen von $\Re_{K}$ und $\Re_{k}$ bezeichen wir mit $h_{K}$ 
bzw. $h_{k}$. Da die Diskriminante von $k$ gleich $-3 q$ ist mit einer Primzahl $q \equiv 1(\bmod 12)$, ist bekanntlich $\mathfrak{\Re}_{k}$ zyklisch und von der Einsgruppe verschieden. Darüber hinaus ist nach einem bekannten Satz von Rédei $h_{k}$ durch 4 teilbar. ${ }^{6)}$

Bezeichnet man mit $M / k$ die größte unverzweigte, abelsche Erweiterung vom 2-Potenzgrade, so ist die Galoisgruppe von $M / k$ zu $\Re_{k}$ isomorph. Da $\Re_{k}$ zyklisch ist, ist die Klassenzahl von $M$ prim zu 2. ${ }^{7)} \quad$ Da aber $K / k$ unverzweigt ist, ist also $h_{K}=h_{k} / 2 \geqq 2$ und $\AA_{K}$ zyklisch. Es gibt daher genau einen unverzweigten, quadratischen Charakter $\psi$ von $K$. Insbesondere ist die einzige unverzweigte, quadratische Erweiterung von $K$ galoissch über $k$.

Man sieht ferner unter Benutzung der Normbildung leicht ein, daß man durch die Einbettung von $k$ in $K$ einen Homomorphismus von $\Re_{k}$ auf $\Omega_{K}$ erhält, dessen Kern von der Ordnung 2 ist.

Die Primteiler $\mathfrak{p}$ in $k$ von in (4) auftretenden Primzahlen $p$ sind träge in $K / k$. Dann sind die in (4) auftretenden Primzahlen gerade diese, deren Primteiler $\mathfrak{p}$ in $k$ in einer erzeugenden Klasse von $\mathfrak{\Omega}_{k}$ enthalten sind. Folglich gehört $p$ auch in $K$ in einer erzeugenden Klasse von $\Re_{K}$ an. Dies bedeutet, daß $p$ in $K$ keiner Quadratklasse der absoluten Idealklassengruppe von $K$ angehört.

Wir nehmen jetzt an, daß es einen relativzyklischen Körper $L / K$ vom dritten Grade mit dem Führer $f(L / K)=(1-\omega)^{e} \mathfrak{p}$ gibt, der sogar über $k$ galoissch ist. Wäre $L / k$ abelsch, so gäbe es in $L / k$ einen Zwischenkörper $K^{\prime} / k$ dritten Grades mit $L=K K^{\prime}$. Wegen $p=N_{k / q}(\mathfrak{p})$ $\not \equiv 1(\bmod 3)$ wäre aber $\mathfrak{p}$ in $K^{\prime} / k$ unverzweigt und so entgegen der Voraussetzung auch in $L / K$. Daher ist $L / k$ nichtabelsch. Dann trifft nur die andere Eventualität, daß die Galoisgruppe $G(L / k)$ isomorph ist zur Diedergruppe $D_{3}$ mit den erzeugenden Relationen $\sigma^{3}=1, \tau^{2}=1, \tau \sigma=$ $\sigma^{2} \tau$. $G(L / k)$ ist daher eine Frobeniusgruppe, deren Frobeniuskern $G(L / K)$ ist. Die beiden nichttrivialen Charaktere $\chi$ ersten Grades von $G(L / K)$ induzieren auf $G(L / k)$ denselben irreduziblen Charakter. Der Artinsche Führer $f(\chi, L / K)$ ist nichts anders als $f(L / K)$. Für die mit Hilfe von diesem $\chi$ und $\psi$ gebildete $L$-Funktion $L(s, \psi \chi)$ in $K$ gilt also nach Satz 1 die Behauptung in Satz 2.

\footnotetext{
6) Für den genannten Satz in der allgemeinen Fassung vergleiche man Fröhlich [4].

7) Dies beweist man leicht unter Benutzung des in Iwasawa [10] geschehenden Gedankengangs.
} 
2.2. Wir müssen noch nur zeigen, daß es wirklich $L / K$ mit den oben genannten Eigenschaften gibt.

Es gibt in $K$ zwei Primteiler $\mathfrak{l}_{1}$ und $\mathfrak{l}_{2}$ von 3 , und $K$ enthält eine primitive 3-te Einheitswurzel. Da die Klassenzahl von $k$ zu 3 prim angenommen ist, ist $\mathfrak{p}$ auch in $K$ äquivalent der 3-ten Potenz eines Ideals in $K$. Nach Zusatz 1 in [11], §3 gibt es dann zwei nichtnegative, ganzrationale Zahlen $e(1)$ und $e(2)$ derart, daß das Ideal $\mathfrak{\Upsilon}_{1}^{e(1)} \mathfrak{r}_{2}^{e(2)} \mathfrak{p}$ in $K$ Führer eines relativzyklischen Körpers $L^{\prime} / K$ vom Relativgrade 3 ist.

Wir bezeichnen nun mit $N$ den kleinsten $L^{\prime}$ umfassenden galoisschen Körper über $k$. $N / K$ ist vom Grade 3 oder $3^{2}$. Ferner bezeichnen wir mit $G(N / K)$ und $G(K / k)=\{1, S\}$ die Galoisgruppen von $N / K$ bzw. $K / k$. Bezeichnet man mit $Z_{3}$ den Ring der ganzrationalen 3-adischen Zahlen, so operiert der Gruppenring $Z_{3}[G(K / k)]$ natürlicherweise auf $G(N / K)$. Man setze $I=(1+S) / 2$ und $J=(1-S) / 2$. Dann ist $G(N / K)$ das direkte Produkt von $G(N / K)^{I}$ und $G(N / K)^{J}$. Den Invariantenkörper von $G(N / K)^{J}$ bezeichnen wir mit $L^{\prime \prime}$ und diesen von $G(N / K)^{I}$ mit $L$. Die beiden genannten Körper sind galoissch über $k ; L^{\prime \prime} / k$ ist abelsch, und die Galoisgruppe $G(L / k)$ ist isomorph zur Diedergruppe $D_{3}$, wenn nur $L \neq K$ ist.

Um $L \neq K$ zu zeigen, sei zunächst $L^{\prime \prime}=K$. Dann ist $N=L=L^{\prime}$ $\neq K$. Zweitens sei $L^{\prime \prime} \neq K$. Dann ist $\mathfrak{p}$, wie in $\S 2.1$ gesehen ist, in $L^{\prime \prime} / K$ unverzweigt. Da aber $N=L^{\prime \prime} L=L^{\prime \prime} L^{\prime}$ ist, ist $\mathfrak{p}$ notwendig in $L / K$ verzweigt. Daher ist auch in diesem Fall $L \neq K$.

In den beiden Fällen ist also $G(L / k)$ isomorph zu $D_{3}$, und der Führer $\mathfrak{f}(L / K)=\mathfrak{f}(\chi, L / K)$ ist von der Form $\mathfrak{Y}_{1}^{e(1)} \mathfrak{r}_{2}^{e(2)} \mathfrak{p}$ mit geeigneten $e(1)$ und $e(2)$. Da aber $L / k$ galoissch ist, bleibt $\mathrm{f}(L / K)$ unter der Wirkung der Galoisgruppe $G(K / k)$ invariant. Somit ist $f(L / K)=(1-\omega)^{e} \mathfrak{p}$ mit einem nichtnegativen, ganzrationalen Exponenten $e$.

Zusammen mit dem in $\S 2.1$ Gesagten ist der Beweis von Satz 2 erledigt.

\section{LITERATUR}

[1] J. V. Armitage, Zeta functions with a zero at $s=1 / 2$, Inventiones math. 15 (1972), 199-205.

[2] E. Artin, Zur Theorie der L-Reihen mit allgemeinen Gruppencharakteren, Hamb. Abh. 8 (1930), 292-306.

[ 3 ] R. Brauer, On the zeta-functions of algebraic number fields, Amer. J. Math. 69 (1947), 243-250. 
[4] A. Fröhlich, The generalization of a theorem of L. Rédei's, Quarterly J. Math. (2), 5 (1954), 130-140.

[5] — Artin-root numbers and normal integral bases for quaternion fields, Inventiones math. 17 (1972), 143-166.

[6] A. Fröhlich und J. Queyrut, On the functional equation of the Artin $L$-function for characters of real representations, Inventiones math. 20 (1973), 125-138.

[ 7 ] H. Hasse, Artinsche Führer, Artinsche $L$-Funktionen und Gausssche Summen..., Acta Salmanticensia, Ciencias: Sec. Mat. 1954.

[ 8 ] B. Huppert, Endliche Gruppen I, Springer Verlag, Berlin Heidelberg New York, 1967.

[ 9 ] M. Ishida, On the divisibility of Dedekind's zeta-functions, Proc. Japan Acad. 33 (1957), 293-297.

[10] $\dot{K}$. Iwasawa, A note on class numbers of algebraic number fields, Hamb. Abh. 20 (1956), 257-258.

[11] S.-N. Kuroda, Über den allgemeinen Spiegelungssatz für galoissche Zahlkörper, J. Number Theory 2 (1970), 282-297.

[12] J.-P. Serre, Représentation linéaires des groupes finis, Paris, Hermann, 1967.

[13] — Conducteurs d'Artin des caractères réels, Inventiones math. 14 (1971), 173183.

[14] J. Thompson, Finite groups with fixed-point-free automorphisms of prime order, Proc. Nat. Acad. Sci. USA 45 (1959), 578-581.

Department of Mathematics

College of General Education

University of Tokyo 